\title{
Cardiomyopathie périnatale
}

\author{
Hilary Bews MD, Mahwash Saeed MD, Shuanvgbo Liu MD
}

Citation : CMAJ 2021 November 15;193:E1733. doi : 10.1503/cmaj.210468-f

Voir la version anglaise de l'article ici : www.cmaj.ca/lookup/doi/10.1503/cmaj.210468

1 La cardiomyopathie périnatale (CMPN) se présente avec des symptômes d'insuffisance cardiaque, dont de la dyspnée, de l'orthopnée et de l'œdème des pieds

La cardiomyopathie périnatale se définit comme une insuffiance cardiaque accompagnée d'une fraction d'éjection du ventricule gauche (FEVG) inférieure à $45 \%$ dont les symptômes se manifestent au cours du dernier mois de grossesse et jusqu'à 5 mois après l'accouchement chez une personne sans cardiopathie préalable ${ }^{1,2}$. Les personnes atteintes d'une insuffiance cardiaque progressive ou grave, d'angine ou d'arythmie (chez environ $20 \%$ des patientes) nécessitent un examen et une orientation auprès d'un cardiologue de toute urgence $\mathrm{e}^{1,3}$.

2

L'échocardiographie est critique au diagnostic de la CMPN et à l'exclusion d'autres causes de dysfonction cardiaque

L'échocardiographie est la référence standard pour la quantification de la FEVG et peut mettre en évidence un thrombus ventriculaire gauche qui est causé par une hypercoagulabilité reliée à la grossesse et une hypokinésie du ventricule gauche.

\section{On devrait traiter les patientes pour une insuffisance}

cardiaque accompagnée d'une FEVG réduite, avec d'importantes modifications spécifiques à la grossesse Les diurétiques, les bêtabloquants, l'hydralazine, les nitrates et la digoxine sont sécuritaires pendant la grossesse et devraient être employés dans le traitement de la $\mathrm{CMPN}^{3}$. Les inhibiteurs de l'enzyme de conversion de l'angiotensine et les bloqueurs des récepteurs de l'angiotensine sont tératogènes et contre-indiqués pendant la grossesse ${ }^{3}$. Compte tenu de la haute prévalence des thrombus ventriculaires gauches (10\%-17\%), l'héparine de faible poids moléculaire peut être envisagée en fin de grossesse et jusqu'à 6-8 semaines après l'accouchement lorsque la FEVG est inférieure à une valeur de $30 \%-35 \%$.

\section{La CMPN est associée à une morbidité substantielle et à la mort}

Jusqu'à la moitié des personnes atteintes de CMPN demeureront avec un dysfonctionnement ventriculaire gauche résiduel ${ }^{2,3}$. Si elle se rétablit, la FEVG se normalisera à une valeur supérieure à $50 \%$, souvent dans les 3-6 mois suivant le diagnostic ${ }^{3}$. Les taux estimés de mortalité à long terme ( $>5$ ans) sont de 7\%-20\% aux États-Unis ${ }^{3}$.

\section{Les personnes enceintes présentant des antécédents de CMPN doivent être orientées de toute urgence auprès d'un obstétricien et d'un cardiologue}

Une éventuelle grossesse n'est pas recommandée si la FEVG ne se rétablit pas à une valeur supérieure à $50 \%-55 \%$, puisque la probabilité d'un décès maternel peut alors atteindre entre $25 \%$ et $50 \%$. 5 . Même si le rétablissement est complet, un risque de rechute de $20 \%$ demeure pour les grossesses subséquentes, ce qui devrait être considéré comme étant à haut risque ${ }^{4}$.

\section{Références}

1. Ezekowitz JA, O'Meara E, McDonald MA, et al. 2017 comprehensive update of the Canadian Cardiovascular Society guidelines for the management of heart failure. Can J Cardiol 2017;33:1342-433.

2. Liu S, Zuberi SA, Malik AA, et al. Peripartum cardiomyopathy characteristics and outcomes in Canadian Aboriginal and non-Aboriginal women. Can J Cardiol 2017;33:471-7.

3. Davis MB, Arany Z, McNamara DM, et al. Peripartum cardiomyopathy: JACC state-of-the-art review. J Am Coll Cardiol 2020;75:207-21.

4. Honigberg MC, Givertz MM. Peripartum cardiomyopathy. BMJ 2019;364:k5287.

5. Elkayam U. Risk of subsequent pregnancy in women with a history of peripartum cardiomyopathy. J Am Coll Cardiol 2014;64:1629-36.

\section{Intérêts concurrents : Aucun déclaré.}

Cet article a été révisé par des pairs.

Affiliations : Section de cardiologie, Département de médecine interne, Collège de médecine Max-Rady, Faculté des sciences de la santé Rady, Université du Manitoba, Winnipeg, Man.

Propriété intellectuelle du contenu : Il s'agit d'un article en libre accès distribué conformément aux modalités de la licence Creative Commons Attribution (CC BY-NCND 4.0), qui permet l'utilisation, la diffusion et la reproduction de tout médium à la condition que la publication originale soit adéquatement citée, que l'utilisation se fasse à des fins non commerciales (c.-à-d., recherche ou éducation) et qu'aucune modification ni adaptation n'y soit apportée. Voir : https://creativecommons.org/ licenses/by-nc-nd/4.0/deed.fr.

Correspondance : Hilary Bews, bewsh@myumanitoba.ca 